\title{
Reactive Oxygen Species Registration in Planarian Regeneration
}

\author{
Kh. P. Tiras ${ }^{1,2}$, S. V. Gudkov ${ }^{1,2,3,4}$, V. I. Emelyanenko ${ }^{1,4}$ \& K. B. Aslanidi ${ }^{1}$ \\ ${ }^{1}$ Institute of Theoretical and Experimental Biophysics of Russian Academy of Sciences, Pushchino, Russia \\ ${ }^{2}$ Pushchino State Institute of Natural Sciences, Pushchino, Russia \\ ${ }^{3}$ Institute of General Physics of Russian Academy of Sciences, Moscow, Russia \\ ${ }^{4}$ National Research University of Nizhni Novgorod, Nizhny Novgorod, Russia \\ ${ }^{5}$ Institute of Biological Instrumentation of Russian Academy of Sciences, Pushchino, Russia \\ Correspondence: K. B. Aslanidi, Institute of Theoretical and Experimental Biophysics of Russian Academy of \\ Sciences, Pushchino, Moscow region, 142290, Russia. E-mail: kbaslanidi@gmail.com
}

Received: August 21, 2015 Accepted: September 2, 2015 Online Published: September 24, 2015

doi:10.5539/apr.v7n6p13 URL: http://dx.doi.org/10.5539/apr.v7n6p13

\begin{abstract}
Reactive oxygen species (ROS) are directly involved in cell proliferation, differentiation and apoptosis in a variety of organisms. We studied kinetics of own luminescence induced by changes of ROS in early stages of planarian regeneration. Kinetics of chemiluminescence were measured in intact planarians and the same individuals after decapitation within 15 hours. We analyzed the traumatic fluorescent signal obtained as the difference between kinetics of intact and decapitated planarians. It was found that regeneration is accompanied by changes in the content of ROS correlated with the energy-intensive process in regenerating planarians. Oxidative stress was caused by damage to cell membranes in the dissection of the planarian and it was accompanied by a drop in the intensity of luminescence with a time constant of about 3.6 hours. Phagocytosis of dying cells by neoblasts was accompanied by an increase of the luminescence intensity after $2-3$ hours after decapitation. Neoblast mitosis was described by two maximums of luminescence over 5.1 hours and 8.3 hours after decapitation. For the first time we demonstrated the opportunity of registering the physiological state of pluripotent stem cells at the level of the organism in vivo.
\end{abstract}

Keywords: reactive oxygen species, chemiluminescence, planarian, regeneration, neoblast, apoptosis, phagocytosis, mitosis

\section{Introduction}

Reactive oxygen species (ROS) play a dual role in cells, tissues and organs of living organisms. On the one hand, ROS are involved in many pathological processes, such as neurodegenerative changes, cardiovascular disorders and cancer. On the other hand, ROS are necessary for normal life, in particular, ROS directly participate in the regeneration process. At the same time, certain cells differentiate, dedifferentiate, enter the mitotic cycle or enter into apoptosis and dismissive (Pirotte et al., 2015). Moreover, maximum growth speed of different living systems is determined by individual levels of exogenous ROS (Ivanova et al., 2005). It is shown that mechanisms of regeneration in a variety of animals (fruit flies, hydra, planarians, zebrafish, etc.) have much in common. A mechanical trauma of all of these animals is accompanied by necrosis and/or apoptosis of damaged cells, phagocytosis, proliferation, primary specialization and/or migration of pluripotent stem cells. Damaged cells release ROS that cause an activation of effector cells, MAPK and/or JNK pathways of intracellular signaling (Vriz et al., 2014). Freshwater planarians are the classical model for the study of regeneration and stem cells biology (Elliott \& Alvarado, 2014; Scimone et al., 2013). The role of ROS in the life of planarians is studied very fragmentary. The aim of the study was to investigate kinetics of ROS-induced luminescence in planarians during the regeneration process. It was found that regeneration accompanied by changes in concentration of ROS correlated with energy-intensive processes such as oxidative stress caused by damage to cell membranes during the dissection of planarians, phagocytosis of dying cells and mitosis of neoblasts. Furthermore, our data suggest that mitosis of two populations of neoblasts is divided by time. 


\section{Method}

Asexual strains of the freshwater planarian Dugesia (Girardia) tigrina were used in the current work. Planarians were maintained in aquarium water with hardness $\sim 3-5 \mathrm{mEq} / \mathrm{L}, \mathrm{rH} \approx 6.5$ at $26{ }^{\circ} \mathrm{C}$. They were fed by larvae of Diptera. In the experiment, we use planarians with length of about $10-11 \mathrm{~mm}$ after starving for at least 7 days. Regeneration was caused by amputation of the head of planarian at the level of the "eyes" (Tiras et al., 2015). Size of planarians after amputation was about $8.7 \mathrm{~mm}$. Decreasing of planarian size effected on neoblast population very slightly, because there is just $5-7 \%$ of neoblast in the amputated head.

In each step of the experiment, intact planarians or their fragments were placed in $10 \mathrm{ml}$ of water inside of special polypropylene scintillation cuvettes (Beckman, USA). Chemiluminometer Biotoks 7A-2M (ANO "Engineering Center - Ecology", Russia) was used for measuring the luminescence. The device is equipped with a photomultiplier 9750QB / 1 (9750QB / 1, EMI Electronics LTD, Middlesex, UK) with a spectral sensitivity area of $380-710 \mathrm{~nm}$. Measurements were carried out at a temperature of $26.0 \pm 0.2^{\circ} \mathrm{C}$ and the photon-counting mode with data recording intervals of 1 second. Dependencies that were obtained were smoothed using SigmaPlot 8.0 software with "Smooth 2D Data" function. The difference between luminescence kinetics of intact planarians and the same worms after dissection was used for the analysis of the fluorescent signal caused by an amputation of planarians. The resulting difference curve was analyzed by using a program based on the method of Marquardt (Marquardt, 1963).

\section{Results}

We registered dependence of chemiluminescence of $10 \mathrm{ml}$ of pure water (Curve 1), 30 specimens of intact planarians of the same size which were placed in $10 \mathrm{ml}$ of pure water (Curve 2) and the same 30 individuals after decapitation which were placed in $10 \mathrm{ml}$ of pure water (Curve 3) (Figure 1). Continuous registration of luminescence was 15 hours. Analysis of dependencies allows us to conclude that the level of registered chemiluminescence of water is an average of 10-11 pulses per second. Against this background, all recorded kinetics demonstrate an attenuation of chemiluminescence during the time of registration:

1). Changes of chemiluminescence of pure water varies slightly over time.

2). Signal of chemiluminescence registered on 30 intact planarians placed in $10 \mathrm{ml}$ of pure water is almost 2 times higher than the signal registered in pure water and decreased by almost $20 \%$ in 15 hours.

3). Signal of chemiluminescence registered on 30 decapitated planarians placed in $10 \mathrm{ml}$ of pure water is about 2 times higher than the signal registered in pure water and decreased by more than $40 \%$ in 15 hours.

Results allow us to identify various sources of chemiluminescence.

Curve 1 in Figure 1 describes chemiluminescence of water; it is presented in all derived kinetics. It is known that the source of free radicals in pure water is dissolved molecular oxygen. In pure water saturated with atmospheric air at the temperature used in the experiment $\left(26^{\circ} \mathrm{C}\right)$ the formation of reactive oxygen species occurs (Bruskov et al., 2002; Gudkov et al., 2012).

Curve 2 in Figure 1 describes chemiluminescence of water and intact planarians. The difference between kinetics 1 and 2 defines changes of chemiluminescence of intact planarians. It is known that the main source of radicals in normally-functioning cells is the reaction of one-electron reduction of molecular oxygen, leading to the formation of superoxide anion radical. A superoxide anion radical is formed by the NADPH oxidative complex of cell membranes: the cytoplasmic membrane, the endoplasmic reticulum membrane and the inner membrane of mitochondria (Pirotte et al., 2015; Vladimirov \& Proskurina, 2009). In all cases, an activation of NADPH-oxidant complex is associated with a functional load and a depolarization of relative membranes, especially at the development of oxidative stress. The second source of free radicals in living cells is a nitrogen monoxide NO, formed by different NO-synthetases. According to various estimates, $2 \%$ to $30 \%$ of consumed oxygen is (normally) converted into ROS in an organism. Increased functional load, in particular motor activity, leads to a sharp increase in the production of ROS. It is necessary to note that under bright light, planarians were active during the selection and placement into scintillation cuvettes, which led to an increase in ROS, and after being placed in the dark measuring camera of the luminometer they were settled down and detectable chemiluminescence level that gradually falls off. 


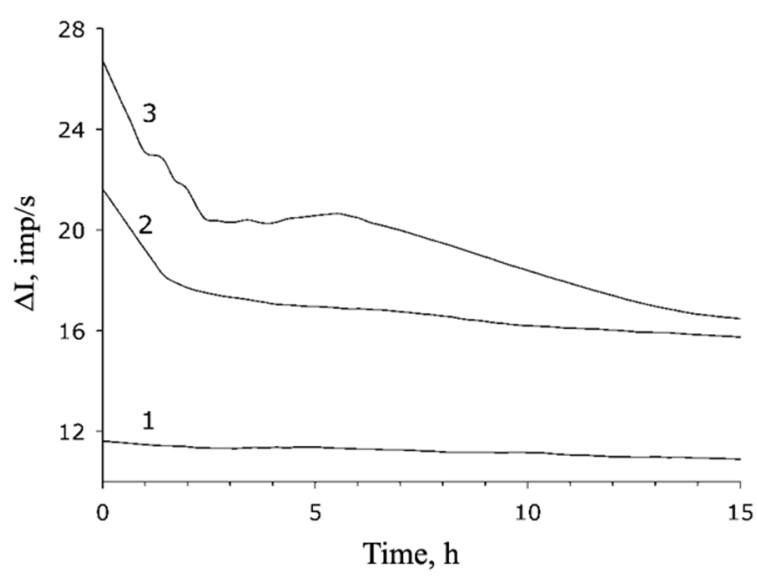

Figure 1. Kinetics of the chemiluminescence

Vertical - chemiluminescence intensity I, pulses/sec, horizontal - time, hours. 1-registered chemiluminescence of $10 \mathrm{ml}$ of pure water, 2-registered chemiluminescence of 30 specimens of intact planarians that were placed in 10 $\mathrm{ml}$ of pure water, 3-the same 30 individuals after decapitation that were placed in $10 \mathrm{ml}$ of pure water.

Curve 3 in Figure 1 describes chemiluminescence of water and decapitated planarians. The difference between kinetics 3 and 2 defines changes of chemiluminescence caused by trauma. In decapitated planarians, in comparison with intact planarians, the number of sources of free radicals increases dramatically. Disturbance of plasma membranes resulted in the development of oxidative stress, which is usually manifested in the accumulation of oxidation products of lipids, proteins, and damaged DNA bases and in reduced levels of antioxidants and increased sensitivity of membrane lipids and lipoproteins to pro-oxidants, including ions $\mathrm{Fe}^{2+}$ or $\mathrm{H}_{2} \mathrm{O}_{2}$ (Pirotte et al., 2015; Vladimirov \& Proskurina, 2009). Moreover, chemiluminescence could arise in the interaction of a protein, or individual amino acids, with peroxynitrite (NO). The steady value of chemiluminescence in injured planarians also significantly exceeded corresponding values in intact animals and clean water. That means that trauma activates mechanisms of regeneration, the duration of which exceeds the duration of the "calm" after normal motor activity registered in intact planarians after being placed in scintillation cuvettes.

\section{Discussion}

Results suggest that the intensity of chemiluminescence defines the functional state of planarians in scintillation cuvettes (Pirotte et al., 2015). Moreover, the fact that up to $30 \%$ of the planarian biomass are neoblasts activated after injury (González- Estévez \& Saló, 2010) suggests that kinetics of ROS-induced chemiluminescence registered in our experiments are largely determined by physiological activity of these pluripotent stem cells.

Thus, based on the analysis of curves in Figure 1, we can conclude that the intensity of neoblast chemiluminescence of decapitated planarians (Curve 3 ) is significantly higher than luminescence of intact animals (Curve 2) and emission of water (Curve 1). That allows us to analyze activity of cellular mechanisms that are triggered immediately after injury. A post-traumatic luminescent signal in Figure 2 was obtained as the difference of kinetics of intact planarians (Figure 1. Curve 2) and the same animals after decapitation (Figure 1. Curve 3). Parameters of decaying exponential curves and three Gaussian distributions were determined by using a program based on the method of Marquardt (Marquardt D.W., 1963), minimizing functional:

$$
S=\left(I_{\text {exp }}-I(t)\right)^{2},
$$

where $I_{\exp }$ - experimentally obtained difference in kinetics of chemiluminescence, I $(t)$ - function describing the difference of kinetics, $t$ - time after placing a prepared in a cuvette of a luminometer. The difference between kinetics of chemiluminescence presented in Figure 2 is described by the function:

$$
I(t)=A_{0} \exp \left(-t / t_{0}\right)+B+\sum_{i} A_{i} \exp \left(-\ln 2\left(\left(t_{i}-t\right) / \sigma_{i}\right)^{2}, i=1,2,3,\right.
$$

$i=1,2,3$, where $A_{0}$ - initial value of the luminescence intensity, $\mathrm{t}_{0}$ - damping time constant, $\mathrm{B}$ - settled value $\mathrm{A}_{\mathrm{i}}$ maximum value, $\mathrm{t}_{\mathrm{i}}$ - maximum position, $2 \sigma_{\mathrm{i}}$ - the half-width of the normal distribution.

$$
\Delta \mathrm{I}=4.45 \exp ^{-0.277 \mathrm{t}}+0.62 \text {, }
$$






Figure 2. The difference between kinetics of chemiluminescence injured and intact planarians

Vertical - the difference between kinetics of chemiluminescence $\Delta \mathrm{I}$, pulses/sec, horizontal - time hours. 1-the difference between kinetics of chemiluminescence obtained in the experiment; 2-the sum of four individual selected functions; 3-decaying exponential $\Delta \mathrm{I}=4.45 \exp ^{-0.277} \mathrm{t}+0.62$; 4-normal distribution $\Delta \mathrm{I}=1.16 \exp (-\ln 2$ $((\mathrm{t}-1.63) / 0.41)^{2} ; 5$-normal distribution $\Delta \mathrm{I}=1.41 \exp \left(-\ln 2((\mathrm{t}-5,11) / 1.85)^{2} ; 6\right.$-normal distribution of $\Delta \mathrm{I}=1.78$ $\exp \left(-\ln 2((\mathrm{t}-8.26) / 3.21)^{2}\right.$. The value of the standard deviation between the experimental and calculated curve does not exceed $1.5 \%$.

Curve 3 in Figure 2, described by a decaying exponential (3) shows that the intensity of chemiluminescence, caused by trauma to the planarian, fell in our experiments with a time constant of about 3.6 hours. It happened due to the radiative deactivation of unstable peroxide, formed by oxidation of various organic substrates in dying and injured cells (Pirotte et al., 2015). Indeed, mechanical injury associated with impaired planarians plasma membranes of cells surrounding wound surface, leakage of potassium ions in the extracellular environment and depolarization of intact muscle cells, lead to a contraction of muscles and wound closure in a few minutes (Baguñà et al., 1998; Almuedo-Castillo, 2014). Moreover, the presence of cell-cell contacts allows depolarization, which spreads to a distance of $0.5 \mathrm{~mm}$ (Aslanidi et al.1996). Considering that the width of experimental planarians (length about $10 \mathrm{~mm}$ ) does not exceed $1.0 \mathrm{~mm}$, and any cross-sections of planarians cause damage of the nerve trunks which extend along the entire body, it can be expected that trauma leads to the spread of depolarization waves throughout planarian bodies. Depolarization, spread through gap junctions from mechanically damaged cells, leads to changes in the ionic composition of intact cells. In particular, the intracellular activity of sodium and calcium is increased and concentration of intracellular potassium is decreased (Potapova et al., 1990; Aslanidi et al., 1991). Evolving oxidative stress induces specific activation of gene JNK by stress-activated protein kinase (SAPK), which belongs to the large family of mitogen-activated protein kinases (MAPKs). The JNK kinase modulates expression of genes associated with trauma, particular induces apoptotic cell death (Almuedo-Castillo, 2014). It should be noted that the development of oxidative stress occurs in the same ways, regardless of its cause (Aslanidi et al., 1997). At the cellular level, oxidative stress induces locomotor activity. Increasing of intracellular calcium ion concentration triggers exocytosis and formation of adjunct. Neoblasts begin to move to damaged cells (Sheimann \& Sakharova, 1974).

Curve 4 in Figure 2, described by normal distribution (4)

$$
\Delta I=1.16 \exp (-\ln 2(t-1.63) / 0.41)^{2}
$$

with maximum of about 1.6 hours, corresponds evidently with process of phagocytosis. Indeed, morphological methods showed that the fragments of dead cells phagocytosed by nearby neoblasts for $1-3 \mathrm{~h}$ (González-Estévez \& Saló, 2010). The difference in the time of maximum activity of phagocytosis is easily explained by the different temperature conditions of experiments (Tiras et al., 2015). It has long been known that phagocytic activity is accompanied by significant changes in the conductivity of the plasma membrane for potassium and hydrogen ions. Phagocytosis is accompanied by a strong increase in the non-selective conductivity of plasma membrane, leading to complete depolarization (Holevinsky \& Nelson, 1998). Depolarization activates power-consuming mechanisms of active ion transport, and as already mentioned, increased functional load leads to a sharp increase in the generation of ROS. Chemiluminescence analysis traditionally has been used to investigate the functional activity 
of phagocytic cells. Absorption of objects of phagocytosis correlated with the generation of oxygen radicals in phagocytic cells and causes enhanced chemiluminescence. During phagocytosis, the cell produces ROS, including superoxide anion radical, hydroxyl anion, singlet oxygen, hydrogen peroxide, and hypochlorite. The process of phagocytosis, in particular by neutrophils of peripheral blood, takes approximately 1 hour (Vladimirov \& Proskurnina, 2009). Note that autophagy is widespread in the animal world and it is an evolutionarily-conserved adaptation to the adverse conditions of an microenvironment (Galluzzi et al., 2014). Phagocytosis in planarians is an essential tool not only for regeneration but also for nourishment. After feeding, the planarian intestinal wall breaks down and contents of the intestine permeate into parenchyma. Intestinal and other cells of the parenchyma (except nerve) phagocytose food, and then the original structure of the body is restored (Sheimann \& Sakharova, 1974). Previously it has been shown that the process of phagocytosis associated with the digestion of food is accompanied by intense chemiluminescence, developing during the day and continuing up for five days (Tiras et al., 2001). In contrast with these experiments, the present study of chemiluminescence of regeneration was carried out with hungry animals, which led to a significant reduction in the overall level of ultra-weak luminescence compared to the phagocytosis after feeding. Nevertheless, it is certain that in both cases, ultra-weak luminescence associated with phagocytosis was registered.

Curves 5 and 6 in Figure 2, described by a normal distributions $(5,6)$

$$
\Delta I=1,41 \exp \left(-\ln 2((t-5.11) / 1.85)^{2}\right.
$$

and

$$
\Delta I=1.78 \exp \left(-\ln 2((t-8.26) / 3.21)^{2},\right.
$$

have a maximum at 5.1 hours and 8.3 hours after decapitation. We can assume that these peaks correspond to a different population of miotic activity neoblasts. Indeed, numerous experiments have shown that the first peak of neoblast proliferation is observed at 6 hours after surgery (Almuedo-Castillo et al., 2014). Some discrepancies of times of maximum miotic activity can be linked with different temperature conditions of experiments. Another experiments revealed two x-ray-sensitive fractions of planarian neoblasts that differed by DNA content and size of cytoplasm (Higuchi et al., 2007). The first fraction contains cells in S/G2/M phases; there were undifferentiated neoblasts and neoblasts in their primary differentiation. The second fraction contains cells in G0/G1 phases; they were a special type of stem cells with small size, several chromatoid bodies, and nuclei enriched by heterochromatin. These populations may include mitosis with time shift.

Another explanation of results connected with the fact that Girardia tigrina is a mixoploid species (Ribas, Riutort, \& Baguna, 1989), i.e. a part of cells of that species, including neoblast, is diploid and a half is triploid, i.e. contains a triple set of chromosomes. It can be assumed that these cell populations are separated in time of DNA synthesis and-will give two peaks, times of occurrence of which will vary in a half. Indeed, in our experiments, the ratio of times of the attaining maximum of mitotic activity from the moment of decapitation was 8.26/5.11=1.61 $\approx 1.5$.

Thus, for the first time we have shown the possibility of registering the physiological state of pluripotent stem cells at the level of the organism in vivo. This possibility is caused by the fact that pluripotent stem cells - neoblasts constitute a significant part of the biomass of planarians (up to 30\% of total cells) (Baguñà et al., 1988). Thus, neoblasts' phagocytic activity, as well as mitosis, activate breathing and increase oxygen consumption and hence stimulate the generation of ROS. Motor activity, developed by planarians while they were caught and placed into the scintillation cuvette, and muscle contraction, leading to wound closure within a few minutes after decapitation, also stimulate the production of ROS. Origin of two peaks of proliferation of neoblast after decapitation must still be clarified and quantification of neoblast involved in the process of regeneration is a goal for the future researches.

\section{Acknowledgments}

This work was supported by grant from RFBR №15-29-01135 and State Task of Ministry of Education and Science of Russian Federation, № 2912. Authors express their sincere gratitude to prof. V.I. Bruskov for helpful comments during the discussion of results.

\section{References.}

Almuedo-Castillo, M., Crespo, X., Seebeck, F., Bartscherer, K., Salò, E., \& Adell, T. (2014). JNK controls the onset of mitosis in planarian stem cells and triggers apoptotic cell death required for regeneration and remodeling. PLOS Genetics, 11(4). http://dx.doi.org/10.1371/journal.pgen.1005132 
Aslanidi, K. B., Aslanidi, G. V., Vachadze, D. M., Zinchenko, V. P., Labas, Yu. A., \& Potapova, T. V. (1997). A possible role of cold-induced ionic stress in cold-induced cell death. Membrane and Cell Biology, 11(1), 57-76.

Aslanidi, K. B., Boitzova, L. J., Chailakhyan, L. M., Kublik, L. N., Marachova, I. I., Potapova, T. V., \& Vinogradova, T. A. (1991). Energetic cooperation via ion-permeable junctions in mixed cell cultures. FEBS Letters, 283(2), 295-297. http://dx.doi.org/10.1016/0014-5793(91)80612-7

Aslanidi, K. B., Vachadze, D. M., Zamyatnin, A. A., Pozharskaya, T. R., Rochev, Y. A., Selezneva, I. I., ... Chailakhyan, L. M. (1996). Compartmentation determines dynamics of multicellular system's growth. Membrane and Cell Biology, 13(3), 289-298.

Baguñà, J., Saló, E., Collet, J., Auladell, M. C., \& Ribas, M. (1988). Cellular, molecular and genetic approaches to regeneration and pattern formation in planarians. Progress in Zoology, 36, 65-78. http://dx.doi.org/10.1007/s00018-007-7426-y

Bruskov, V. I., Karp, O. E., Garmash, S. A., Shtarkman, I. N., Chernikov, A. V., \& Gudkov, S. V. (2012). Prolongation of oxidative stress by long-lived reactive protein species induced by X-ray radiation and their genotoxic action. Free Radical Research, 46, 1280-1290. http://dx.doi.org/10.3109/10715762.2012.709316

Bruskov, V. I., Malakhova, L. V., Masalimov, Z. K., \& Chernikov, A. V. (2002). Heat-induced formation of reactive oxygen species and 8-oxoguanine, a biomarker of damage to DNA. Nucleic acids research, 30(6), 1354-1363. http://dx.doi.org/10.1093/nar/30.6.1354

Elliott, S. A., \& Alvarado, A. S. (2013). The history and enduring contributions of planarians to the study of animal regeneration. Wiley interdisciplinary reviews. Developmental Biology, 2(1), 301-326. http://dx.doi.org/10.1002/wdev.82

Galluzzi, L., Pietrocola, F., Levine, B., \& Kroemer, G. (2014). Metabolic control of autophagy. Cell, 159(6), 1263-1276. http://dx.doi.org/10.1016/j.cell.2014.11.006

González-Estévez, C., \& Saló, E. (2010). Autophagy and apoptosis in planarians. Apoptosis, 15(3), 279-292. http://dx.doi.org/10.1007/s10495-009-0445-4

Gudkov, S. V., Bruskov, V. I., Astashev, M. E., Chernikov, A. V., Yaguzhinsky, L. S., \& Zakharov, S. D. (2011). Oxygen-dependent auto-oscillations of water luminescence triggered by the $1264 \mathrm{~nm}$ radiation. The Journal of Physical Chemistry B., 115(23), 7693-7698. http://dx.doi.org/10.1021/jp2023154

Gudkov, S. V., Ivanov, V. E., Karp, O. É., Chernikov, A. V., Belosludtsev, K. N., Bobylev, A. G., ... Bruskov, V. I. (2014). Impact of biologically important anions on reactive oxygen species formation in water under the effect of non-ionizing physical agents. Biofizika, 59(5), 862-870. http://dx.doi.org/10.1134/s00063509 $1405008 x$

Gudkov, S. V., Karp, O. E., Garmash, S. A., Ivanov, V. E., Chernikov, A. V., Manokhin, A. A., .. Bruskov, V. I. (2012). Generation of reactive oxygen species in water under exposure of visible or infrared irradiation at absorption band of molecular oxygen. // Biofizika, 57(1), 5-13. http://dx.doi.org/10.1134/s0006350 912010113

Higuchi, S., Hayashi, T., Hori, I., Shibata, N., Sakamoto, H., \& Agata, K. (2007). Characterization and categorization of fluorescence activated cell sorted planarian stem cells by ultrastructural analysis. Development, Growth \& Differentiation, 49(7), 571-581. http://dx.doi.org/10.1111/j.1440-169x.2007.00947.x

Holevinsky, K. O, \& Nelson, D. J. (1998). Membrane capacitance changes associated with particle uptake during phagocytosis in macrophages. Biophysical Journal, 75(5), 2577-2586 http://dx.doi.org/10.1016/s0006-3495 (98)77703-3

Ivanova, A. E., Aslanidi, K. B., Karpenko, I. V., \& Belozerskaia, T. A. (2005). The effect of hydrogen peroxide on the growth of microscopic mycelial fungi isolated from habitats with different levels of radioactive contamination. Microbiology, 74(6), 655- 663. http://dx.doi.org/10.1007/s11021-005-0120-x

Marquardt, D. W. (1963). An algorithm for least-square estimation of nonlinear parameters. Journal of the Society for Industrial and Applied Mathematics, 11(2), 431-441. http://dx.doi.org/10.1137/0111030

Pirotte, N., Stevens, A. S., Fraguas, S., Plusquin, M., Van Roten, A., Van Belleghem, F., ... Smeets, K. (2015). Reactive Oxygen Species in Planarian Regeneration: An Upstream Necessity for Correct Patterning and Brain Formation. Medicine and Cellular Longevity, 2015, 1-19. http://dx.doi.org/10.1155/2015/392476 
Potapova, T. V., Aslanidi, K. B., \& Boitzova, L. J. (1990). Energy transfer via cell-to-cell junction: Ouabain-resistant cells maintain a membrane potential in ouabain-sensitive cells. FEBS Letters, 262(1), 69-71. http://dx.doi.org/10.1016/0014-5793(90)80156-d

Ribas, M., Riutort, M., \& Baguna, J. (1989). Morphological and biochemical variation in populations of Dugesia (G.) tigrina (Turbellaria, Tricladida, Paludicola) from the western Meditterranean: biogeographical and taxonomical implications. Journal of Zoology, 218(4). 609-626. http://dx.doi.org/10.1111/j.1469-7998.1989. tb05003.x

Scimone, L. M., Kravarik, K. M., Lapan, S. W., \& Reddien, P. W. (2014). Neoblast Specialization in Regeneration of the Planarian Schmidtea mediterranea. Stem Cell Reports, 3(2). 339-352. http://dx.doi.org/10. 1016/j.stemcr.2014.06.001

Sheimann, I. M., \& Sakharova, N. J. (1974). On a peculiarity of planariandigestion. Comparative Bicochemistry and Physiology, 48, 601-607.

Tiras, K. P., Petrova, O. N., Myakisheva, S. N., Popova, S. S., \& Aslanidi, K. B. (2015). Effects of weak magnetic fields on different phases of planarian regeneration. Biophysics, 60(1), 126-130. http://dx.doi.org/10.1134/ s0006350915010224

Tiras, K., Novikov, C., \& Voeykov, V. (2001). Ultra-weak irradiation of planarians: a new model for the study phagocytosis. Proceedings of IX International Symposium of the Biology of Turbellaria. Barcelona, 50.

Vladimirov, Y. A., \& Proskurnina, U. V. (2009). Free radicals and cell chemiluminescence. Biochemistry (Moscow), 74(13), 1545-1566. http://dx.doi.org/10.1134/s0006297909130082

Vriz, S., Reiter, S., \& Galliot, B. (2014). Cell death: a program to regenerate. Current Topics in Developmental Biology, 108, 121-51. http://dx.doi.org/10.1016/b978-0-12-391498-9.00002-4

\section{Copyrights}

Copyright for this article is retained by the author(s), with first publication rights granted to the journal.

This is an open-access article distributed under the terms and conditions of the Creative Commons Attribution license (http://creativecommons.org/licenses/by/3.0/). 